\title{
A Biochemical Blueprint for Long-Term Memory
}

\author{
Erik D. Roberson and J. David Sweatt ${ }^{1}$ \\ Division of Neuroscience \\ Baylor College of Medicine \\ Houston, Texas 77030-3498 USA
}

\begin{abstract}
The greatest barrier to the long-term storage of information in a biological system is the inevitability of molecular turnover. In this review, we discuss the features required of any chemical mechanism capable of overcoming this obstacle, positing that a specific type of "mnemogenic", or memory-forming, chemical reaction is the basis of the engram. We describe how molecules as diverse as protein kinases, prions, and transcription factors can participate in mnemogenic reactions, and outline a blueprint for memory that postulates mnemogenic reactions at the synapse and in the nucleus and considers the constraints imposed by requirements for high fidelity and the ability to forget. This sort of a priori analysis may facilitate directed experimental approaches to understanding the mechanisms of lifelong memory.
\end{abstract}

\section{Introduction}

Recent decades have witnessed rapid progress in understanding the molecular and cellular basis of learning and memory in a variety of organisms. As yet, however, no system of long-term information storage in the central nervous system has been dissected experimentally to the point of identifying how it overcomes its greatest threat: molecular turnover. Consider a group of classmates at a high school reunion recalling names, stories, and places they had not pondered in 25 years; how were those memories faithfully stored in brains constructed with molecular building blocks that in the

${ }^{1}$ Corresponding author. interim had been degraded and replaced probably hundreds of times?

Our goal in this review is to present a deductive analysis of the necessary organization of any long-term memory system that successfully defeats turnover of its constituent components, with the belief that such an analysis will both enable a directed search for the appropriate elements and provide a standard against which any proposed solution to the memory problem can be measured. The foundation of our blueprint for memory is a specific type of chemical reaction, referred to as the mnemogenic reaction based on the Greek for memory forming. We assert that this type of chemical reaction is the only means by which information storage in biological systems can be rendered immune to molecular turnover. After formulating the mnemogenic chemical reaction and describing various forms that such a reaction can take, the discussion will consider in less formal terms the subcellular loci in which such reactions are required for long-term memory, the mechanisms needed to maintain high fidelity, and how these memories can be forgotten. Finally, in the last section we refer to two generic alternatives to our model; information storage without molecular turnover and activity-dependent dynamic stabilization of neuronal circuits.

Before proceeding, two points merit clarification. First, it is apparent that the model we propose does not apply to the many forms of shorter term memory, even those lasting hours or days, that do not require protection from molecular turnover. Second, there is significant evidence that structural changes may be involved in many models of learning and memory, and perhaps because of the seemingly inherent stability of "structure," the suggestion now appears with some frequency in the literature that memory could be "either biochemical or structural." We believe that this is an inappropriate dichotomy, because clearly any cellular structure is composed of molecules subject to con-

LEARNING \& MEMORY 6:381-388 @ 1999 by Cold Spring Harbor Laboratory Press ISSN1072-0502/99 \$5.00

$$
\begin{array}{llllllllllllllll}
L & E & A & R & N & I & N & G & \underset{381}{\mathbf{Z}} & M & E & M & O & R & Y
\end{array}
$$


stant degradation and replacement and thus still requires a mechanism to ensure that it will be reconstructed appropriately by the new building blocks; we argue that this mechanism is a mnemogenic chemical reaction.

\section{The Mnemogenic Chemical Reaction}

At the heart of this discussion is the postulate that a biological memory mechanism capable of lasting a lifetime will have as its hallmark a mnemogenic chemical reaction. Specific examples of reactions that qualify as mnemogenic were independently proposed in the mid-1980s by Crick (1984) and Lisman (1985; see below). The basic and most general formulation of the mnemogenic reaction is described by equation 1 .

$$
X+X^{\bullet} \rightarrow X^{\bullet}+X^{\bullet}
$$

In this reaction, $X$ is a bistable species that can exist in either of two states, a basal state $(X)$ or an activated or modified form $\left(X^{\bullet}\right)$. Learning invokes an initiator mechanism causing the activation of species $\mathrm{X}$, that is; the conversion of a molecule of $X$ into the $X^{*}$ form.

$$
X \stackrel{\text { initiator }}{\longrightarrow} X^{\bullet}
$$

This activated $X^{\bullet}$ leads, directly or indirectly, to manifestation of the memory phenotype, presumably but not necessarily by impinging on the process of synaptic transmission. The fundamental feature of the mnemogenic reaction is that in the presence of the activated molecule, $X^{\bullet}$, an inactive molecule of $X$ is converted to the $X^{\bullet}$ form generating a positive feedback loop. This is the means by which levels of $X^{\bullet}$ are sustained despite molecular turnover; while the cell synthesizes the molecule only in the inactive form, the activated species catalyzes activation of the nascent species, and thus is able to propagate itself through generations of turnover.

Chemical interactions that meet the criteria for a mnemogenic reaction have been described in a variety of biological systems. These examples can be divided into groups based on the nature of the interaction between the $X$ and $X^{\bullet}$ species (see also Lisman and Fallon 1999). Some of the specific examples may not be easily envisioned playing a role in learning and memory; our goal here is merely to appreciate the variety of forms the mnemogenic reaction may take.

\section{COVALENT MODIFICATION MNEMOGENIC REACTIONS}

In some of the initial work to appear in the literature concerning memory and molecular turnover, Crick (1984) described and Lisman (1985) formalized a scheme for defeating molecular turnover that can be arranged as a mnemogenic reaction where $X^{\bullet}$ is a covalently modified form of $X$. Shortly thereafter, the exciting discovery was made that calcium/calmodulin-dependent protein kinase II (CaMKII) could participate in just such a reaction (Saitoh and Schwartz 1985; Lai et al. 1986; Lou et al. 1986; Miller and Kennedy 1986; Schworer et al. 1986). CaMKII, which has become the archetypal example of what we term a covalent modification mnemogenic reaction, is synthesized in the inactive state and triggered to undergo autophosphorylation by calcium and calmodulin; once this occurs, the phosphorylated form of CaMKII is autonomously active, even in the absence of the calcium/ calmodulin initiator. Importantly, the inactive $(X)$ form of CaMKII is a substrate for the phosphorylated $\left(X^{\bullet}\right)$ form of CaMKII, in a mnemogenic phosphorylation reaction that occurs between subunits in a CaMKII holoenzyme.

$$
\text { CaMKII + CaMKII-P } \rightarrow \text { CaMKII- } P+\text { CaMKII-P }
$$

A nascent molecule of CaMKII arriving in an environment where the kinase had been autonomously activated could be inserted into a holoenzyme and phosphorylated by other, active subunits, thereby becoming active itself; the autonomous activity of CaMKII would thus be maintained through cycles of protein turnover.

To effectively serve as mnemogenic, such covalent modification reactions must conform to two mechanistic requirements that are necessary for one molecule of $X\left(X^{\bullet}\right)$ to be able to alter the state of another molecule of $X$. First, it should be clear that only enzymes undergoing intermolecular autocatalysis can participate in a mnemogenic reaction; intramolecular autocatalysis does not suffice. Second, in the case of enzymes with multisubunit holoenzymes, the holoenzyme must not be synthesized and degraded en bloc, but rather must undergo subunit exchange; that is, it must turn over by serial replacement of individual subunits so that nascent molecules can interact with existing, active ones. Importantly, in the case of CaMKII, intermolecular autophosphorylation has been dem-

$$
\begin{array}{lllllllllllllll}
L & E & A & R & N & I & N & G & \begin{array}{l}
\boldsymbol{Z} \\
\mathbf{3 8 2}
\end{array} & M & E & M & O & R & Y
\end{array}
$$


onstrated but the required subunit exchange remains unproved experimentally.

Covalent modification mnemogenic reactions need not be based on phosphorylation. For example, an autocatalytic glycosyltransferase or ADPribosyltransferase could function similarly. Alternatively, instead of small molecules, the reaction could involve addition or removal of elections in a redox reaction. In addition, there are known examples of covalent modification mnemogenic reactions involving proteolysis; activated trypsin protease $\left(X^{\circ}\right)$ can cleave its inactive zymogen, trypsinogen $(X)$, to yield another molecule of active trypsin.

\section{CONFORMATIONAL MNEMOGENIC REACTIONS}

A second type of mnemogenic reaction is based on a structural or conformational interaction between $X$ and $X^{\bullet}$. The exemplar for this class is the prion protein, $\mathrm{PrP}$, a neuronal protein involved in infectious spongiform encephalopathies such as scrapie (see Prusiner 1998). PrP is a bistable molecule that can exist either in the cellular form $\left(\operatorname{PrP}^{\mathrm{C}}\right)$ in which it is synthesized by neurons or the scrapie form $\left(\mathrm{PrP}^{\mathrm{Sc}}\right)$ associated with encephalopathy. $\operatorname{PrP}^{\mathrm{Sc}}$ is a post-translationally modified variant of $\operatorname{PrP}^{\mathrm{C}}$ believed to possess an altered conformation. A fascinating aspect of $\mathrm{PrP}$ is that it undergoes a mnemogenic reaction in which $\operatorname{PrP}^{\mathrm{Sc}}\left(X^{\bullet}\right)$ can induce the conversion of $\operatorname{PrP}^{\mathrm{C}}(X)$ into new $\operatorname{PrP}^{\mathrm{Sc}}$.

$$
\mathrm{PrP}^{\mathrm{C}}+\mathrm{PrP}^{\mathrm{Sc}} \rightarrow \operatorname{Prp}^{\mathrm{Sc}}+\operatorname{Prp}^{\mathrm{Sc}}
$$

By this means, the prion effects a type of memory whereby the infection is maintained despite turnover of the PrP.

It appears that this type of mnemogenic reaction may also occur in physiologic situations. Recent research has demonstrated that the yeast elements [PSI+] and [URE3], which impart specific metabolic phenotypes, represent specific conformations of the proteins Sup35 and Ure2, respectively, and are inherited in a dominant, nonchromosomal manner (see Lindquist 1997). In fact, these elements are passed on to progeny in the cytoplasm and convert nascent Sup35 and Ure2 into $[P S I+]$ and $[U R E 3]$ through a prionlike conformational reaction.

\section{SYNTHETIC MNEMOGENIC REACTIONS}

A third category of mnemogenic reactions is based on the synthesis of the species involved, rather than on postsynthetic modifications of the species as in the first two groups. The classic example of this type of reaction is autoregulation of the bacteriophage $\lambda$ repressor (Ptashne et al. 1976). When $\lambda$ infects a bacterium, it enters a lysogenic phase until induced to enter its lytic cycle. This lysogenic phase is sustained by $\lambda$ repressor, which prevents transcription of the genes required for lysis. Adequate levels of $\lambda$ repressor are maintained in spite of protein turnover by virtue of the fact that the repressor binds DNA sequences in the regulatory region upstream of its gene and stimulates its own transcription, which after translation leads to production of the repressor. In the context of the mnemogenic reaction, $X$ is the pool of amino acid building blocks necessary to synthesize the repressor and $X^{\bullet}$ is a fully formed repressor.

$$
\text { amino acids }+\lambda \operatorname{Rep} \rightarrow \lambda \operatorname{Rep}+\lambda \operatorname{Rep}
$$

Examples of this type of autoregulatory mnemogenic reaction are also found in eukaryotes. MyoD, a transcription factor that directs the differentiation of muscle cells, stimulates its own synthesis (Thayer et al. 1989), maintaining a "memory" of the fact that the cell has entered its specified lineage. The mec-3 gene of Caenorbabditis elegans, a homeobox gene involved in the differentiation of certain touch receptors, also positively regulates its own synthesis (Way and Chalfie 1989). A variation on this theme of chemical species that promote their own synthesis is DNA itself, which serves as a template to direct its own replication.

\section{CIRCULAR MNEMOGENIC REACTIONS}

In each of the examples we have considered, the $\mathrm{X}^{\bullet}$ species directly feeds back on $X$ to promote formation of additional $X^{\bullet}$. Indirect, circular mechanisms are also possible; for example, $X^{\bullet}$ could lead to activation of species $Y$, yielding $Y^{\bullet}$,

$$
X^{\bullet}+Y \rightarrow X^{\bullet}+Y^{\bullet}
$$

whereas $Y^{\cdot}$ in turn interacts with $X$ to convert it to $X^{\text {. }}$.

$$
X+Y^{\bullet} \rightarrow X^{\bullet}+Y^{\bullet}
$$

The sum of these two partial reactions

$$
X+X^{\bullet}+Y+Y^{\bullet} \rightarrow X^{\bullet}+X^{\bullet}+Y^{\bullet}+Y
$$

demonstrates that this type of circular cascade produces a series of stably activated species $\left(X^{\bullet}, Y^{\bullet}\right.$, etc.), one or more of which could serve as the

$$
\begin{array}{lllllllllllllll}
\hline & E & A & R & N & I & N & G & \mathbf{Z} & M & E & M & 0 & R & Y
\end{array}
$$


effector of the memory phenotype. An example of this type of reaction system was proposed as a potential mechanism for memory (Lynch and Baudry 1984), whereby activated calpain protease $\left(X^{\bullet}\right)$ would lead to "activation" of glutamate receptors in the form of a greater receptor density $\left(Y \rightarrow Y^{\bullet}\right)$, whereas the increase in receptors $\left(Y^{\bullet}\right)$ would stimulate activation of calpain $\left(X \rightarrow X^{\bullet}\right)$, producing a persistent increase in both calpain activity and glutamate receptor density. A recent, formal analysis has demonstrated that multiple different kinase cascades that alone do not participate in covalent modification mnemogenic reactions can, using crosstalk, function collectively as a circular mnemogenic reaction (Bhalla and Iyengar 1999).

\section{Subcellular Loci of Mnemogenic Reactions}

Although a mnemogenic chemical reaction is necessary for information storage, a single such reaction may not be sufficient. In fact, on the basis of morphological features of a neuron, one can predict that at least two are required.

\section{NUCLEAR LOCUS}

The mnemogenic reaction is a robust mechanism for information storage outlasting turnover of individual molecules, but it is not fail-safe. In particular, it is vulnerable to a transient interruption in the supply of species $X$. If no new $X$ is produced during the lifetime of $X^{\bullet}$, the reaction will fail; even if synthesis of $X$ resumes, there would be no extant $X^{\bullet}$ to activate it. To preclude this problem, a mnemogenic reaction must be imposed in the nucleus to ensure sufficient production of species $X$. This is likely to be the sort of synthetic mnemogenic reaction described above and involving a self-reinforcing transcriptional activator to sustain the supply of $X$.

There are two basic strategies by which this nuclear mnemogenic reaction might be initiated. First, each new learning event could cause a signal to be sent to the nucleus, sparking synthesis of species $X$ (Goelet et al. 1986); at first blush, this dynamic modulation appears to be an efficient solution, because the energy expenditure required to carry on the nuclear mnemogenic reaction would be linked to the need for it at the synapse. However, when one considers the likelihood that new learning events may occur quite frequently and assumes that the memory mechanism is conserved from synapse to synapse (i.e., that there is not a separate $X$ for each synapse), it seems redundant to reinduce this reaction with each new episode of learning. The alternative, and more parsimonious, strategy would be to initiate the reaction once, at or before the very first episode of learning in the history of the cell. In this case, the initiation of the nuclear reaction would be tantamount to a step in the differentiation of the cell into a neuron capable of supporting memory storage and would henceforth produce a constitutive supply of $X$.

Our discussion thus far has had a theoretical focus. However, it is worthwhile to try to relate the theoretical consideration of this concept of a nuclear mnemogenic reaction to current experimental work on hippocampal long-term potentiation (LTP). There is considerable evidence that the induction of long-lasting LTP is associated with alterations in gene expression (Mackler et al. 1992; Impey et al. 1996). Similar observations that changes in gene expression are associated only with long-term memories have been made in a variety of systems, and the consistency of this observation suggests that the new gene products may be involved in a mnemogenic reaction. A common assumption is that the new gene product is species $X$, but we have just argued that it is unlikely that synthesis of species $X$ is reinduced each time a memory is stored. ${ }^{1}$ The alternative possibility is that the gene products transcribed de novo as a result of LTP induction do not constitute species $X$, but rather the initiator of Equation 2; unlike species $X$, the initiator may have a relatively short halflife and require reinduction each time a memory destined for lifelong storage is created. In this scheme, the initiator of the mnemogenic reaction is not a calcium transient or second messenger, but a new gene product. In terms of timing, during the interval in which the initiator is being synthesized and transported back to the synapse, other impermanent mechanisms that do not require a mnemogenic reaction (e.g., E-LTP) would support the potentiation; only several hours after LTP induction would the mnemogenic reaction be initiated. One

\footnotetext{
${ }^{1}$ However, there is one scenario in which the new gene products could represent species $X$. When a large proportion of the cell's synapses are simultaneously potentiated, e.g., by an LTP-inducing stimulus, the basal level of species $X$ could be intensified to meet the high demand. In this case, signaling to the nucleus would stimulate amplification of an already-initiated mnemogenic reaction.
}

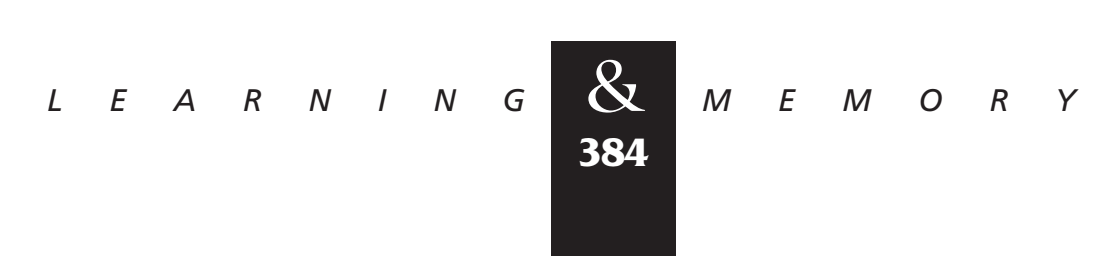


advantage of this hypothesis is that requiring the signal for initiation of the energetically costly mnemogenic reaction to tap the nucleus for a new gene product separates it mechanistically from induction of the more common, shorter-term memory storage systems, thus decreasing the likelihood of inadvertent initiation.

\section{SYNAPTIC LOCUS}

Although the nucleus is likely to be one site of a mnemogenic reaction, at a minimum serving to maintain a constant supply of species $X$, we also predict that the nucleus will not be the only site for a mnemogenic reaction in the neuron. A solely nuclear locus would be insufficient to support contemporary models of memory that postulate that the fundamental unit of information storage in the brain is the synapse, and that the many thousands of synapses onto a single neuron can be modulated independently. This idea is supported by studies of activity-dependent changes in synaptic efficacy such as LTP, which have demonstrated that these changes can be "synapse specific" (Andersen et al. 1977; Lynch et al. 1977). The requirement for synapse specificity leads to the prediction that a mnemogenic reaction will be found at the synapse. The activated product $\left(X^{\bullet}\right)$ of this synaptic reaction could serve in either of two roles. In the simple case, the synaptic $X^{\bullet}$ serves as the species fostering increased synaptic strength, that is, as the potentiating species. Another possibility is that the potentiating species is produced by a mnemogenic reaction at an extra-synaptic site, for example, in the soma. If this were the case, there must be some means of ensuring that the potentiating species is transported only to potentiated synapses; therefore, these synapses must have some sort of tag to identify them (Lisman 1995; Sossin 1996; Frey and Morris 1997). To survive protein turnover, this tag must be the product of a mnemogenic reaction. Thus, even if the potentiating species is produced extrasynaptically, a synaptic $X^{\bullet}$ is required to serve as a resident tag.

\section{Proofreading for High Fidelity}

An additional prediction arises from consideration of the tremendous importance of maintaining localization of the mnemogenic reaction to the potentiated synapse. If a molecule of $X^{\bullet}$ were to diffuse to an adjacent, unpotentiated synapse, it would initiate activation of the inactive $X$ molecules in that synapse, leading to inappropriate potentiation and a loss of fidelity in information storage. Therefore, mobility of the species involved in the synaptic mnemogenic reaction is likely to be constrained, for example, by anchoring to cytoskeletal elements or by a diffusion barrier in the spine neck.

No such mechanism can be completely infallible, however, and over the years for which a very long-lasting memory is stored, even a slow rate of release of $X^{\bullet}$ would pose problems. Therefore, one can predict that the neuron will contain some type of editing function, whereby isolated molecules of $X^{\bullet}$ are inactivated. For example, if $X$ is activated by phosphorylation, as with CaMKII, an editing function could be provided by filling the cell with a low level of phosphatase activity, sufficient to inactivate a small amount of $X^{\bullet}$ drifting out of a potentiated synapse, but insufficient to defeat the mnemogenic reaction within the domain of the synapse, where $X^{\bullet}$ levels are high. It has been demonstrated that in such a system, as long as levels of $X^{\bullet}$ remain above a certain threshold the mnemogenic reaction will continue, whereas where $X^{\bullet}$ is below threshold, as it would be outside the synapse after the leak of a molecule of $X^{\bullet}$, the mnemogenic reaction will be terminated (Lisman 1985). Thus, an editing function can preserve the fidelity of memory storage, even when mechanisms for anchoring of the mnemogenic reaction are imperfect.

\section{Biochemical Mechanisms of Forgetting}

The mnemogenic reaction hypothesis leads to interesting predictions regarding the nature of forgetting. Of course, many memories are not destined for lifelong storage and will not be committed to a mnemogenic reaction; these memories will be short-lived by nature. But even long-lasting memories can be forgotten, and at least three mechanisms can be envisioned. First, the memory may be erased passively due to failure of the mnemogenic reaction, for example, if the flow of nascent species $X$ is interrupted long enough for all of the $X^{\bullet}$ to be degraded. This sort of passive mechanism is likely to be a stochastic process. Second, in cases where it is desired that a memory be forgotten, it may be actively erased. This could be effected by activation of an enzyme that catalyzed the inactivation of $X^{\bullet}$.

$$
\begin{array}{lllllllllllllll} 
& E & A & R & N & I & N & G & \begin{array}{r}
\boldsymbol{Q} \\
385
\end{array} & M & E & M & O & R & Y
\end{array}
$$




$$
X \cdot \stackrel{\text { eraser }}{\longrightarrow} X
$$

Third, a memory may be forgotten even in cases when the mnemogenic reaction perseveres if the mechanism for retrieval of the memory malfunctions. In this case, the memory itself is preserved, but cannot be accessed.

\section{Assumptions and Alternatives}

We have argued that long-lasting memory in a biological system must be subserved by mnemogenic chemical reactions. Of course, any such proposition is based on a series of assumptions, and it is worthwhile to delineate the premises underlying the hypothesis and consider the alternative possibilities should they not prove valid.

One assumption is that all of the chemical species comprising a neuron are subject to turnover. Obviously the alternative possibility is that the molecules responsible for storing the memory do not turn over, but rather are preserved for the lifetime of the memory (Crick 1984, Lisman and Fallon 1999). For a molecule to be maintained in an active state without degradation for years seems rather unlikely, but it has been suggested that in the immune system, follicular dendritic cells may maintain indefinitely pieces of foreign antigen to stimulate memory $B$ cells after the source of antigen has disappeared (see Tew et al. 1980). As yet, such isolation from turnover has not been demonstrated.

A second assumption underlying the hypothesis is that molecular turnover is a stochastic process, that is, any given molecule of $X$ is equally likely to be degraded. The alternative possibility is that molecules somehow age, and "older" molecules are more likely to be degraded than "younger" ones. If this were the case, a variant form of the mnemogenic reaction could explain memory, in which the activation is handed off from older molecules destined for destruction to newer molecules.

$$
X_{\text {new }}+X_{\text {old }}^{\bullet} \rightarrow X_{\text {new }}^{\bullet}+X_{\text {old }}
$$

It is also assumed that neurons do in fact possess an intrinsic mechanism whereby the problem of molecular turnover is defeated. The fact that several different types of mnemogenic reactions have been demonstrated in biology seems to support this premise, but the alternative is that memory is actually not rendered immune to molecular turn- over by intrinsic biochemical processes. If this were the case, any memory destined to be stored for longer than the lifetime of the relevant molecules would have to be regularly relearned by a process of rehearsal. Thus, a mnemogenic reaction (equation 1) would not be required to maintain $X^{\bullet}$, because the serial rehearsal would serve to periodically reinitiate the conversion of $X$ to $X^{\bullet}$ (equation 2). Although it seems that there are at least some cases in which memory can be stored for years without rehearsal, as in the classic example of riding a bike, the possibility that memory is only sustained past the lifetime of individual molecules by relearning cannot be discounted completely. In particular, one variation on the rehearsal model that does not require literal relearning of the information is the dynamic stabilization model (Kavanau 1997; Horn et al. 1998). In this model periodic reactivation of the synapses storing information leads to stabilization of their synaptic weights within a memory storage circuit, by typical Hebbian mechanisms. Interestingly, the periodic reactivation in many variants of this model occurs during sleep.

Finally, it should be formally stated that the basis of memory is assumed to reside in changes in the function of neurons, for example, changes in synaptic strength. The other possibility is the now discounted reverberating circuit model, which postulated that memory is stored by the serial, electrical activation of a population of neurons anatomically organized to feed back onto itself. It is interesting to note that memory storage by the reverberating circuit can be fashioned as a mnemogenic reaction at a nonchemical level. Here it is a cell-biological level, where an activated neuron rather than an activated molecule serves to reactivate itself, in this case through a circuit of other neurons.

\section{Generality of the Mnemogenic Reaction}

We have promulgated in this review the idea that the basis of long-lasting memory storage in the brain is a specific type of chemical reaction. This mnemogenic reaction is the means by which a neuron defeats the problem of molecular turnover. It is argued that memory storage requires, at a minimum, a mnemogenic reaction at potentiated (or depotentiated) synapses, a mnemogenic reaction in the nucleus to ensure sufficient production of

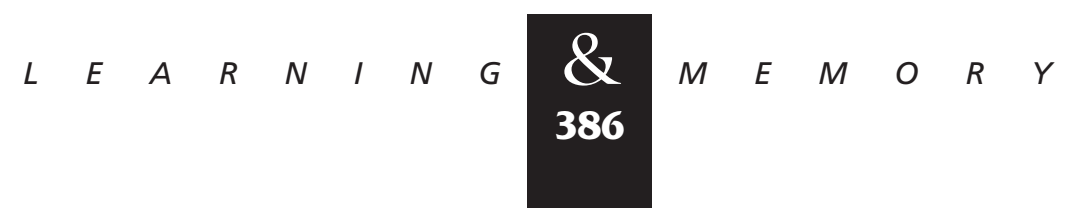


reactants for the synapse, and an editing function to provide for high fidelity.

The discussion has emphasized the role of the mnemogenic reaction in neuronal memory storage. However, examples in which the mnemogenic reaction is the basis for other types of biological memory have also been considered. One example is the mnemogenic reaction involving $\lambda$ repressor, by which a virus remembers to not yet lyse its host. Another classic example of biological memory is differentiation. How does a cell remember for its entire lifetime what developmental lineage it has entered? By mnemogenic reactions, MyoD ensures that once a mammalian cell begins the process of differentiating into a myoblast, it remembers its commitment to that lineage, and mec3 ensures that a worm's touch receptors continue to express their characteristic features. Even the very process by which a cell remembers its own genetic information, DNA replication, is a mnemogenic chemical reaction. Thus, the mnemogenic reaction is a general chemical descriptor of biological information storage.

The question of how organisms store critical information for prolonged periods in spite of constant molecular turnover is one of the most intriguing questions in biology. In cases where this puzzle has been solved, the mnemogenic chemical reaction lies at the heart of the answer. For neuroscientists interested in the basis of long-lasting memory, the challenge is to identify species $X$.

\section{Acknowledgments}

We thank Arnold Eskin and an anonymous reviewer for helpful suggestions and criticisms. We are also grateful to many of our colleagues at Baylor College of Medicine, particularly Joey English, Saurabh Sinha, Jim Lamphear, John Assad, Ron Davis, Dan Johnston, and Paul Pfaffinger, for insightful discussions and comments on early versions of the manuscript.

The publication costs of this article were defrayed in part by payment of page charges. This article must therefore be hereby marked "advertisement" in accordance with 18 USC section 1734 solely to indicate this fact.

\section{References}

Andersen, P., S.H. Sundberg, O. Sveen, and H. Wigström. 1977. Specific long-lasting potentiation of synaptic transmission in hippocampal slices. Nature 266: 736-737.

Bhalla, U.S. and R. Iyengar. 1999. Emergent properties of networks of biological signaling pathways. Science 283: 381-387.

Crick, F. 1984. Memory and molecular turnover. Nature 312: 101.
Frey, U. and R.G.M. Morris. 1997. Synaptic tagging and long-term potentiation. Nature 385: 533-536.

Goelet, P., V.F. Castellucci, S. Schacher, and E.R. Kandel. 1986. The long and the short of long-term memory-a molecular framework. Nature 322: 419-422.

Horn, D., N. Levy, and E. Ruppin. 1998. Memory maintenance via neuronal regulation. Neural Computation 10: $1-18$.

Impey, S., M. Mark, E.C. Villacres, S. Poser, C. Chavkin, and D.R. Storm. 1996. Induction of CRE-mediated gene expression by stimuli that generate long-lasting LTP in area CA1 of the hippocampus. Neuron 16: 973-982.

Kavanau, J.L. 1997. Memory, sleep, and the evolution of mechanisms of synaptic efficacy maintenance. Neuroscience 79: 7-44.

Lai, Y., A.C. Nairn, and P. Greengard. 1986. Autophosphorylation reversibly regulates the $\mathrm{Ca}^{2+} /$ calmodulin-dependence of $\mathrm{Ca}^{2+} /$ calmodulin-dependent protein kinase II. Proc. Natl. Acad. Sci. 83: 4253-4257.

Lindquist, S. 1997. Mad cows meet Psi-chotic yeast: The expansion of the prion hypothesis. Cell 89: 495-498.

Lisman, J.E. 1985. A mechanism for memory storage insensitive to molecular turnover: A bistable autophosphorylating kinase. Proc. Natl. Acad. Sci. 82: 3055-3057.

1995. What does the nucleus know about memories? J. NIH Res. 7: 43-46.

Lisman, J.E. and J.R. Fallon. 1999. What maintains memories? Science 283: 339.

Lou, L.L., S.J. Lloyd, and H. Schulman. 1986. Activation of the multifunctional $\mathrm{Ca}^{2+} /$ calmodulin-dependent protein kinase by autophosphorylation: ATP modulates production of an autonomous enzyme. Proc. Natl. Acad. Sci. 83: 9497-9501.

Lynch, G. and M. Baudry. 1984. The biochemistry of memory: A new and specific hypothesis. Science 224: 1057-1063.

Lynch, G.S., T. Dunwiddie, and V. Gribkoff. 1977. Heterosynaptic depression: A postsynaptic correlate of long-term potentiation. Nature 266: 737-739.

Mackler, S.A., B.P. Brooks, and J.H. Eberwine. 1992. Stimulus-induced coordinate changes in mRNA abundance in single postsynaptic hippocampal CA1 neurons. Neuron 9: $539-548$.

Miller, S.G. and M.B. Kennedy. 1986. Regulation of brain type II $\mathrm{Ca}^{2+} /$ calmodulin-dependent protein kinase by autophosphorylation: $\mathrm{A} \mathrm{Ca}^{2+}$-triggered molecular switch. Cell 44: $861-870$.

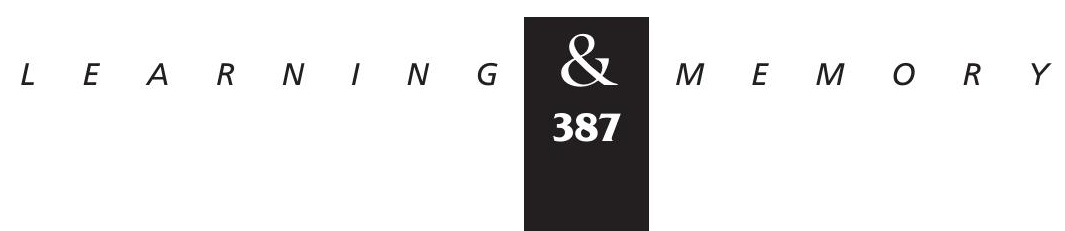




\section{Roberson and Sweatt}

Prusiner, S.B. 1998. Prions. Proc. Natl. Acad. Sci. 95: 13363-13383.

Ptashne, M., K. Backman, M.Z. Humayun, A. Jeffrey, R. Maurer, B. Meyer, and R.T. Sauer. 1976. Autoregulation and function of a repressor in bacteriophage $\lambda$. Science 194: 156-161.

Saitoh, T. and J.H. Schwartz. 1985.

Phosphorylation-dependent subcellular translocation of a $\mathrm{Ca}^{2+} /$ calmodulin-dependent protein kinase produces an autonomous enzyme in Aplysia neurons. J. Cell Biol. 100: $835-842$.

Schworer, C.M., R.J. Colbran, and T.R. Soderling. 1986. Reversible generation of a $\mathrm{Ca}^{2+}$-independent form of $\mathrm{Ca}^{2+}$ (calmodulin)-dependent protein kinase II by an autophosphorylation mechanism. J. Biol. Chem. 261: 8581-8584.

Sossin, W.S. 1996. Mechanisms for the generation of synapse specificity in long-term memory: The implications of a requirement for transcription. Trends Neurosci. 19: 215-218.

Tew, J.G., R.P. Phipps, and T.E. Mandel. 1980. The maintenance and regulation of the humoral immune response: Persisting antigen and the role of folicular antigen-binding dendritic cells as accessory cells. Immunol. Rev. 53: 175-201.

Thayer, M.J., S.J. Tapscott, R.L. Davis, W.E. Wright, A.B. Lassar, and H. Weintraub. 1989. Positive autoregulation of the myogenic determination gene MyoD1. Cell 58: 241-248.

Way, J.C. and M. Chalfie. 1989. The mec-3 gene of Caenorhabditis elegans requires its own product for maintained expression and is expressed in three neuronal cell types. Genes \& Dev. 3: 1823-1833.

Received November 29, 1998; accepted in revised form July 15, 1999. 


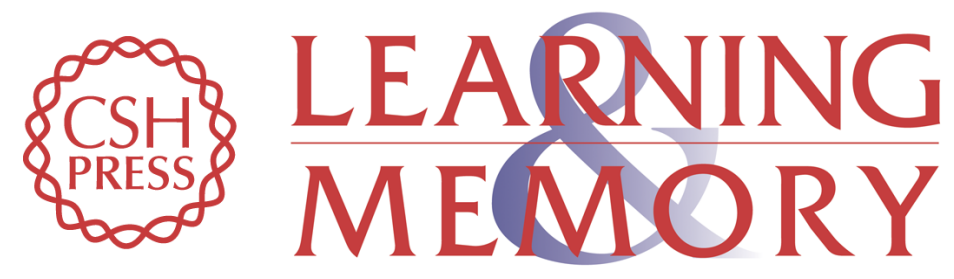

\section{A Biochemical Blueprint for Long-Term Memory}

\section{Erik D. Roberson and J. David Sweatt}

Learn. Mem. 1999, 6:

Access the most recent version at doi:10.1101//m.6.4.381

References This article cites 25 articles, 11 of which can be accessed free at: http://learnmem.cshlp.org/content/6/4/381.full.html\#ref-list-1

License

Email Alerting Receive free email alerts when new articles cite this article - sign up in the box at the Service top right corner of the article or click here. 